\title{
Further Insight into the Reactivity of 3-(p- Chlorophenylimino-methyl) Chromone toward Phosphorus Reagents
}

\section{Maha D. Khidre}

Chemical Industries Division, National Research Centre, Cairo, Egypt.

\begin{abstract}
AERIES of phosphono-substituted and 5-membered ring $A$ chromenes were prepared in reasonable yields from the reactions of 3-(p-chlorophenyl-iminomethyl) chromone (1) with diffrent types of the Wittig-Horner reagents $4 \mathrm{a}-\mathrm{c}, 9$ in the presence of a base. On the other hand by applying cyanomethylenetriphenylphosphonium chloride 12 and allyltriphenylphosphonium bromide 15 to 1 , the corresponding propanenitrile and methylpropenyl chromene derivatives were obtained.
\end{abstract}

Keywords: 3-(Aryliminomethyl) chromone, Wittig-Horner reagents, Phosphonium salts, Phosphono and Substituted chromenes.

In a series of articles from this laboratory, we reported on the synthesis and reactions of new phosphono-substituted heterocycles starting from the inexpensive and easily accessible $\alpha$-phosphonyl carbanions toward different unsaturated systems ${ }^{(1-7)}$.

This article represents an efficient route to the synthesis of phosphono-substituted chromenes and azaphospholylchromene of potential interest to biochemistry and pharmaceutical chemistry. The methodology is based upon the application of $\alpha$ phosphonyl carbanions (Wittig Horner-WH reagents) 4a-c, 9 and phosphonium salts 12, 15 toward 3-(p-chlorophenyl-iminomethyl) chromone (1).

\section{Results and Discussion}

In a previous study, it was reported ${ }^{(8)}$ that chromone (1) underwent a condensation reaction when it was allowed to react with stabilized Wittig reagents $2 \mathrm{a}, \mathrm{b}$ to give the complex ylidenetriphenylphosphorane structure 3 (Scheme 1).

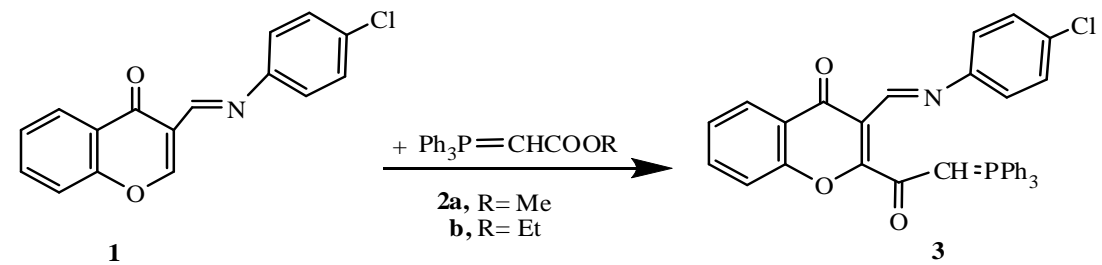

Scheme 1

E-mail: mdkhedr@yahoo.com 
3-(p-Chlorophenyl- iminomethyl) chromone (1) was allowed to react with one equivalent of diethylphosphonoacetates $4 \mathrm{a}$ or $4 \mathrm{~b}$ and diethylcyanomethylphosphonate $4 \mathrm{c}$ in refluxing tetrahydrofuran (THF) containing aqueous $\mathrm{LiOH}$. The product mixture was then subjected to column chromatography to afford the phosphonosubstituted chromenes $5 \mathrm{a}$-c in $\approx 45 \%$ yield and chromene derivatives $8 \mathrm{a}$-c in $\approx 28 \%$ yield (Scheme 2).

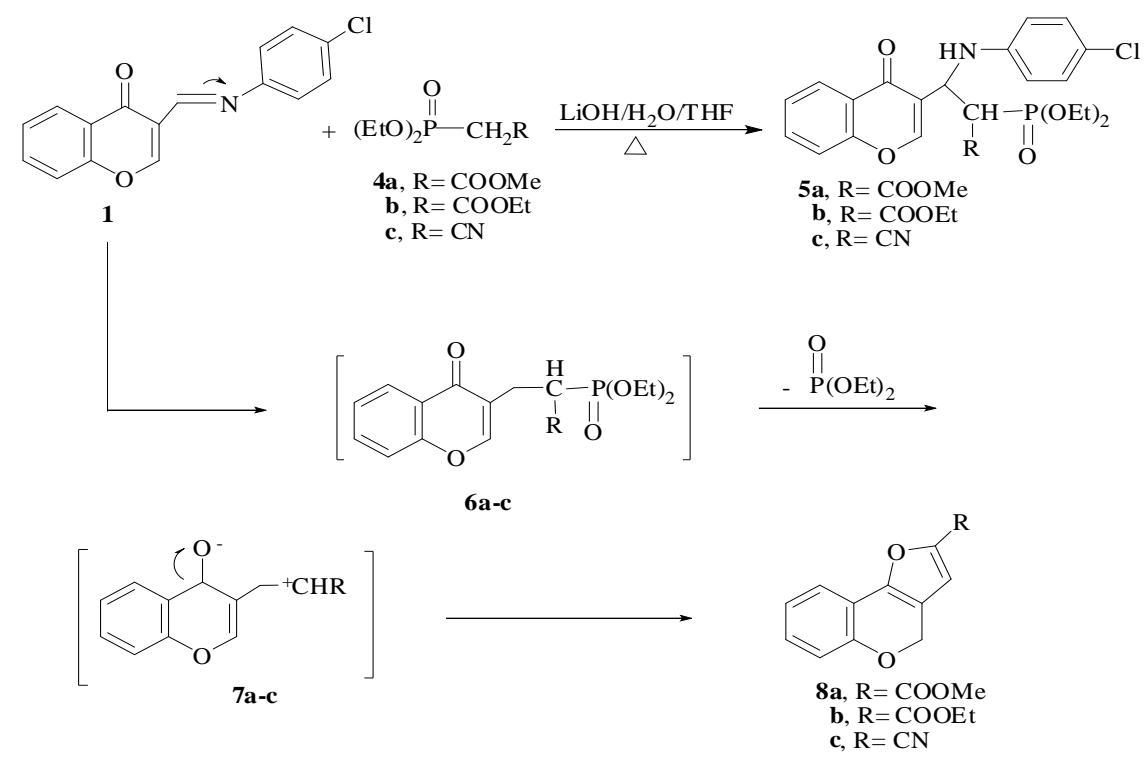

Scheme 2

The chemical structure of 5a was in accord with elemental analysis, molecular weight determination (MS) and spectroscopic data. The ${ }^{31} \mathrm{P}$ NMR spectrum showed a chemical shift $=20.2 \mathrm{ppm}$, that indicates a phosphonate structure $^{(9,10)}$. The IR spectrum of 5a showed absorption bands at $1730(\mathrm{C}=\mathrm{O}$, ester), $3260(\mathrm{NH})$ and $1269(\mathrm{P}=\mathrm{O})$; its ${ }^{1} \mathrm{HNMR}$ revealed the presence of two ethoxy groups attached to phosphorus, the two equivalent $\left[\mathrm{P}\left(\mathrm{OC}_{2} \mathrm{H}_{5}\right)_{2}\right]$ protons coupled with phosphorus appeared as a doublet of triplets (dt, $\mathrm{J}_{H-H}=6.8,{ }^{4} \mathrm{~J}_{H-P}=$ $\left.4.6 \mathrm{~Hz}, 6 \mathrm{H}, 2 \mathrm{x} H_{3} \mathrm{CC} . \mathrm{OP}\right)$ at $1.3 \mathrm{ppm}$ and $4.1\left(\mathrm{dq}, \mathrm{J}_{H-H}=6.8,{ }^{3} \mathrm{~J}_{H-P}=5.5 \mathrm{~Hz}, 4 \mathrm{H}\right.$, $2 \mathrm{x}_{2} \mathrm{COP}$ ) whereas methoxy group appeared as a singlet at $3.7 \mathrm{ppm}$, the spectrum also showed two doublets (each with $=\mathrm{J}_{H-H} 9 \mathrm{~Hz}$ ) due to aromatic protons of the $\mathrm{N}-\mathrm{C}_{6} \mathrm{H}_{4}-\mathrm{Cl}$ moiety $(4 \mathrm{H})$ at 6.6 and 7.1 . The remaining aromatic protons $(5 \mathrm{H})$ appeared as a multiplet in the 7.4-8.3 ppm region. The $\mathrm{NH}$ proton gave a $\mathrm{D}_{2} \mathrm{O}$ exchangeable singlet at 9.19 whereas the exocyclic methine proton $(\mathrm{CH}-\mathrm{N})$ appeared as a doublet of doublet due to coupling with the phosphorus atom at $5.3 \mathrm{ppm}{ }^{3} \mathrm{~J}_{\mathrm{H}-\mathrm{P}}=11.5 \mathrm{~Hz}$, also a doublet of doublet $\left({ }^{2} \mathrm{~J}_{\mathrm{H}-\mathrm{P}}=18 \mathrm{~Hz}\right)$ at 3.33 ppm due to $(\mathrm{CH}-\mathrm{P})$. In the ${ }^{13} \mathrm{CNMR}\left(\mathrm{CDCl}_{3}, \mathrm{ppm}\right)$ spectrum ${ }^{(11)}$ of 5a carbon attached to phosphorus (-CH-P) appeared at $45.7\left(\mathrm{~d},{ }^{1} \mathrm{~J}_{C-P}=147 \mathrm{~Hz}\right)$ while the ($C \mathrm{H}-\mathrm{N})$ located at $53.5\left(\mathrm{~d},{ }^{2} \mathrm{~J}_{C-P}=33 \mathrm{~Hz}\right)$ other signals were displayed at $163.4(\mathrm{~d}$, ${ }^{2} \mathrm{~J}_{C-P}=28, C=\mathrm{O}$, ester $), 185.7\left(\mathrm{~d},{ }^{4} \mathrm{~J}_{C-\mathrm{P}}=4.6, C=\mathrm{O}\right.$, ring).

Egypt. J. Chem. 53, No.5 (2010) 
A reasonable mechanism of the formation of 5a-c might involve an initial nucleophilic ${ }^{(12)}$ attack of the phosphonyl carbanions $4 \mathrm{a}-\mathrm{c}$ on the azomethinecarbon atom in 1 to yield 5a-c. On the other hand, the spectroscopic analyses of the second product clearly demonstrated that the carboxylate products 8a,b, carbonitrile $8 \mathrm{c}$ were formed, The ${ }^{1} \mathrm{HNMR}\left(\mathrm{CDCl}_{3}\right)$ spectrum of $8 \mathrm{~b}$ showed a triplet at $1.3 \mathrm{ppm}$ due to the $\mathrm{CH}_{3} \mathrm{CO}$, ester, $\mathrm{J}_{H-H}=7.5 \mathrm{~Hz}$, also at $4.30 \mathrm{ppm}$ (q, $2 \mathrm{H}, \mathrm{CH}_{2} . \mathrm{O}, \mathrm{J}_{\mathrm{H}-\mathrm{H}}=7.5 \mathrm{~Hz}$ ), whereas the singlet at $5.0 \mathrm{ppm}$ was assigned to the methylene protons. The aromatic protons $(5 \mathrm{H})$ gave a multiplet in the 6.90-7.47 ppm region. In the ${ }^{13} \mathrm{CNMR}\left(\mathrm{CDCl}_{3}\right.$, ppm) spectrum of $8 \mathrm{~b}$ the carboxyl carbon $(C=\mathrm{O})$ appeared at 160.42. According to the mechanism outlined in Scheme 2, the formation of 8a-c could be explained via decomposition of 1 and nucleophilic attack $^{(13)}$ of carbanion center of 4a-c onto the carbon -1, 2-azaphosphol-5-yl]-4Hchromen-4-one (11). According to the mechanism outlined in atom in 1 to afford intermediates $6 \mathrm{a}-\mathrm{c}$. Elimination of a phosphonate moiety and ring closure could explain the formation of the target chromene carboxylate 8a-c.

The behavior of arylimine 1 toward unsaturated phosphonyl carbanion, diethylvinylphosphonate 9 was investigated next. The reaction was carried out under similar reaction conditions, giving 3-[1-(4-chlorophenyl)-2-ethoxy-2-oxido-2, 5dihydro- $1 H$ (Scheme 3), the addition of 9 to 1 gave intermediate 10 , further intramolecular cyclisation of 10 afforded the phosphole 11 via the loss of ethanol molecule ${ }^{(14)}$.

This new reaction led to the formation of the cyclic product 11 . Compound 11 was isolated as pale brown crystals in $68 \%$ yield. The structure seemed possible for the reaction product since the IR $\left(\mathrm{cm}^{-1}\right)$ spectrum of 11 revealed the presence of strong absorption bands at $1248(\mathrm{P}=\mathrm{O})$, and $1098\left(\mathrm{P}_{-}-\mathrm{OC}_{2} \mathrm{H}_{5}\right)$, the ${ }^{31} \mathrm{P}$ NMR spectrum showed a chemical shift $\delta_{P}=12.2 \mathrm{ppm}$. Moreover, the ${ }^{1} \mathrm{H}$ NMR spectrum of compound 11 in $\mathrm{CDCl}_{3}$ gave signals at $1.42 \mathrm{ppm}\left(\mathrm{dt}, \mathrm{J}_{H-H}=6.8,{ }^{4} \mathrm{~J}_{H-P}=\right.$ $4.4 \mathrm{~Hz}, 3 \mathrm{H}, H_{3}$ CC.OP), $3.89\left(\mathrm{dq}, \mathrm{J}_{H-H} 6.8,{ }^{3} \mathrm{~J}_{H-P}=5.6 \mathrm{~Hz}, 2 \mathrm{H}, \mathrm{H}_{2} \mathrm{COP}\right)$ corresponding to phospholyl moiety $[\mathrm{EtOP}(\mathrm{O})]$ and at $5.05\left(\mathrm{~d},{ }^{3} \mathrm{~J}_{H-P}=5.2 \mathrm{~Hz}, 1 \mathrm{H}\right.$, $\mathrm{CH}-\mathrm{N})$ due to methine proton, also at $6.43,6.51\left(2 \mathrm{~d},{ }^{2} \mathrm{~J}_{H-\mathrm{P}}=13.5,1 \mathrm{H}, \mathrm{CH}=\mathrm{CH}-\right.$ $\mathrm{P})$.The remaining aromatic protons $(9 \mathrm{H})$ and $1 \mathrm{H}$ due to $(\mathrm{CH}=\mathrm{CH}-\mathrm{P})$ appeared as a multiplet in the 7.5-8.48 ppm region.<smiles>O=c1c(CNc2ccc(F)cc2)coc2ccccc12</smiles><smiles>O=c1c(C(C=CP(=O)([O-])[O-])Nc2ccc(Cl)cc2)coc2ccccc12</smiles><smiles>CC[14C]([14CH2])=O</smiles>
$\mathrm{LiOH} / \mathrm{H}_{2} \mathrm{O} / \mathrm{THF}$

$-\mathrm{EtOH}$<smiles>C=CC(c1coc2ccccc2c1=O)N(c1ccc(Cl)cc1)P(=O)(OC)OCC</smiles>

11

Scheme 3

Egypt. J. Chem. 53, No.5 (2010) 
On treating arylimine 1 with cyanomethylenetriphenylphosphonium chloride 12 in $\mathrm{THF} / \mathrm{LiOH} / \mathrm{H}_{2} \mathrm{O}$, 3-[(4-chlorophenyl) amino]-3- (4-oxo-4H-chromen-3-yl) propanenitrile (14), 58\% yield was obtained according to Scheme 4. Triphenylphosphine oxide $\left(\mathrm{C}_{6} \mathrm{H}_{5}\right)_{3} \mathrm{P}(\mathrm{O})$ was isolated and identified (TLC) in this reaction. The product 14 had infrared bands at $2200 \mathrm{~cm}^{-1}(\mathrm{CN})$ and $3260 \mathrm{~cm}^{-1}$ attributed to the $(\mathrm{NH})$. In the ${ }^{1} \mathrm{HNMR}$ of 14 the exocyclic proton was shown at 5.19 as a triplet while the methylene protons appeared as two doublets at 3.90, 4.09. The $\mathrm{NH}$ proton gave a $\mathrm{D}_{2} \mathrm{O}$-exchangeable singlet at $9.4 \mathrm{ppm}$. The azomethine-carbon atom at 47.49 and methylene carbon at 23.35 in the ${ }^{13} \mathrm{C}$ NMR spectrum of 14 .
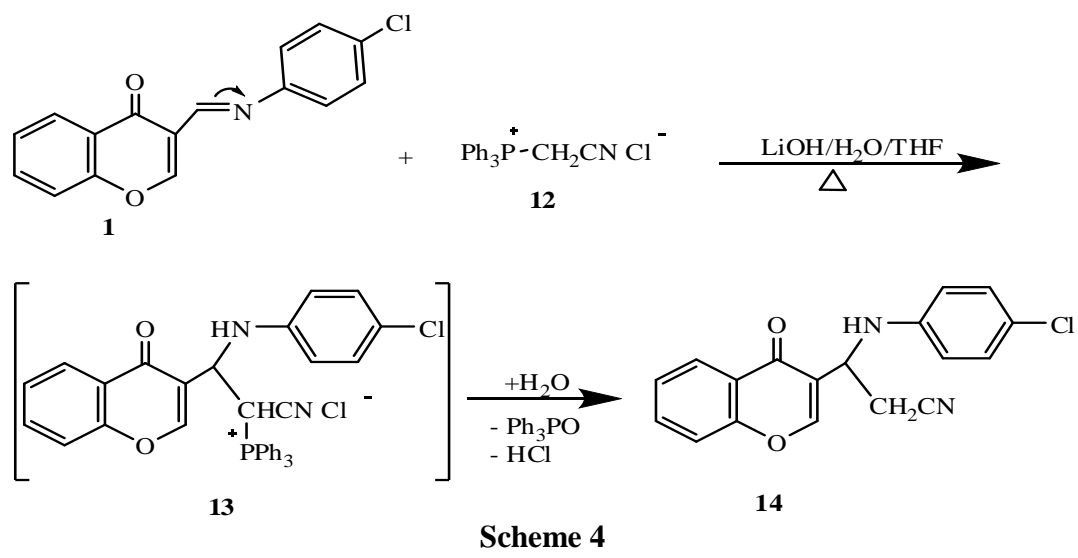

According to Scheme 4, an initial nucleophilic addition of the cabanion center of 12 to the azomethine-carbon atom led to the formation of zwitter ion 13 . Elimination of triphenylphosphine oxide from 13 can lead to the formation of propanenitrile derivative $14^{(15)}$.

Finally, the heating of 1 with one equivalent of allyltriphenylphosphonium bromide 15 in THF under reflux with a catalytic amount of $\mathrm{LiOH} / \mathrm{H}_{2} \mathrm{O}$ gave 3 $\{1$-[(4-chlorophenyl)amino]-2-methylprop-2-en-1-yl $\}-4 H$-chromen-4-one (17), $60 \%$ yield as the sole product (Scheme 5). Obviously, compound 17 is the product of the initial addition 1 to the beta-carbon in 15 , further hydrolysis of 16 and elimination of triphenylphosphine oxide yielded the final product $17^{(16)}$. On the basis of comparative IR, ${ }^{1} \mathrm{HNMR}$, MS and elemental analyses, the structure of compound 17 was deduced ( $c f$. Experimental). 
<smiles>O=c1c(C=Nc2ccc(Cl)cc2)coc2ccccc12</smiles><smiles>CC(C)[C@H](Nc1ccc(Cl)cc1)C(c1ccccc1)c1coc2ccccc2c1=O</smiles>

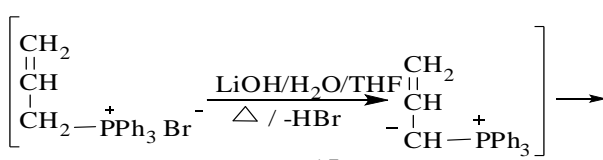

15<smiles>C=C(C)C(Nc1ccc(Cl)cc1)c1coc2ccccc2c1=O</smiles>

Scheme 5

\section{Conclusion}

In view of all the facts previously cited, it can be concluded that 3 -(pchlorophenyliminomethyl)-chromone (1) with Wittig 2a,b, 12, 15 and WittigHorner reagents $4 \mathrm{a}-\mathrm{c}, 9$ led to different products, depending on the nature of the phosphorus reagent used as well as on the stability of the addition products. The results of the previous and the present work point out the variety of the reactions, which can follow an initial attack of phosphorus carbanions onto the azomethine-carbon in 1 . However, the transformations were quite different. The main difference between the present work and the corresponding work of the Wittig reagents with the same substrate 1 is that in the latter case, the transformation of the products was accompanied by elimination of the phosphorus moiety. This was because $\mathrm{Ph}_{3} \mathrm{P}$ species was a much better leaving group than $\left[(\mathrm{EtO}){ }_{2} \mathrm{PO}^{-}\right]$. There was much precedence for this difference. Finally, the present work described an efficient and simple approach to the synthesis of a variety of phosphono-substituted and 5-membered ring chromenes derivatives in reasonable yields. This was achieved by application of the appropriated $\alpha$ phosphonyl carbanions to 1. Data on the pharmaceutical potency as antiinflammatory agents as well as fungicides or antibacterial of the new phosphorylated compounds will be published elsewhere.

\section{Experimental}

\section{General}

Melting points (m.p.) are uncorrected. Infrared sepectra were measured with a Perkin-Elmer IR-spectrometer model 597 using $\mathrm{KBr}$ discs. ${ }^{1} \mathrm{H}$ and ${ }^{31} \mathrm{C}$ NMR spectra were recorded by a Bruker Model WH-270 MHz spectrometer, using TMS as an internal reference. Chemical shifts are given in the $\delta$-scale (ppm), coupling constants $\mathrm{J}$ are given in $\mathrm{Hz} .{ }^{31} \mathrm{P}$ NMR spectra were run on a Varian CFT-20 relative to external $\mathrm{H}_{3} \mathrm{PO}_{4}$. Mass spectra were run at $70 \mathrm{eV}$ on a Schimatzu GCS-QPEX spectrometer provided with a data system. The 
appropriate precautions in handling moisture-sensitive compounds were observed. The silica gel used for column chromatography was Kieselgel 60; particle size 0.2-0.5 mm (E. Merck, Darmstadt).The substrate monoanil 1 was prepared as described by Fitton et $\mathrm{l}^{(17)}$.

Treatment of 3-(p-chlorophenyl-iminomethyl)chromone (1) with $\alpha$-phosphonyl carbanions (4a-c). Preparation of $5 a-c$ and $8 a-c$

General procedure

A solution of $4 \mathrm{mmol}$ of Wittig-Horner reagents $4 \mathrm{a}, 4 \mathrm{~b}$ or $4 \mathrm{c}$ and $1 \mathrm{~g}$ of 1 (3.5mmol) in $30 \mathrm{ml}$ THF was treated with $10 \mathrm{ml}$ of aqueous $\mathrm{LiOH}$ solution $(0.5 \mathrm{M})$. The reaction mixture was stirred at r.t. for $2 \mathrm{hr}$ to ensure the complete dissolve of the reaction then heated under reflux for 6-8 $\mathrm{hr}$ (TLC). After removing the solvent, $20 \mathrm{ml}$ of dist. $\mathrm{H}_{2} \mathrm{O}$ was added and then extracted with $\mathrm{CHCl}_{3}$. After evaporation of the dried $\mathrm{CHCl}_{3}$ solution, the residue was chromatographed on silica gel using n-hexane with increasing amounts of AcOEt as eluents, whereupon compounds 5a-c and 8a-c were isolated.

[2-[(4-chlorophenyl)amino]-1-(methoxycarbonyl) -2-(4-oxo-4H- chromen-3yl) ethyl] diethyl-phosphonate (5a)

5a was obtained $(4: 6, \mathrm{v} / \mathrm{v})$ as pale brown crystals $44 \%$ m.p. $198-200{ }^{\circ} \mathrm{C}$ (from benzene). (Found: $\mathrm{C}, 55.54 ; \mathrm{H}, 4.9 ; \mathrm{Cl}, 7.35 ; \mathrm{N}, 3.0 ; \mathrm{P}, 6.19 . \mathrm{C}_{23} \mathrm{H}_{25} \mathrm{ClNO}_{7} \mathrm{P}$ (493.89), requires C, 55.93; H, 5.10; $\mathrm{Cl}, 7.18 ; \mathrm{N}, 2.84 ; \mathrm{P}, 6.27) ; \mathrm{IR}(\mathrm{KBr}): \widetilde{v}=$ $1640(\mathrm{C}=\mathrm{O}$, ring), $1730(\mathrm{C}=\mathrm{O}$, ester), $1269(\mathrm{P}=\mathrm{O}), 1128(\mathrm{P}-\mathrm{O}-\mathrm{C}), 3260(\mathrm{NH}) \mathrm{cm}$ ${ }^{1} ;{ }^{1} \mathrm{H}$ NMR $\left(\mathrm{CDCl}_{3}\right): \delta=1.3\left(\mathrm{dt}, \mathrm{J}_{H-H} 6.8,{ }^{4} \mathrm{~J}_{H-P} 4.6,6 \mathrm{H}, 2 \times H_{3} \mathrm{CC} . \mathrm{OP}\right), 3.3(\mathrm{dd}, 1 \mathrm{H}$, $\left.{ }^{2} \mathrm{~J}_{H-P} 18, \mathrm{CH}-\mathrm{P}\right), 3.7\left(\mathrm{~s}, 3 \mathrm{H}, \mathrm{CH}_{3} \mathrm{O}\right.$, ester) $4.1\left(\mathrm{dq}, \mathrm{J}_{H-H} 6.8{ }^{3} \mathrm{~J}_{H-P} 5.5,4 \mathrm{H}\right.$, $\left.2 \mathrm{xH}_{2} \mathrm{COP}\right), 5.3\left(\mathrm{dd}, 1 \mathrm{H},{ }^{3} \mathrm{~J}_{H-P} 11.5 \mathrm{CH}-\mathrm{N}\right), 6.6,7.1\left(2 \mathrm{~d}, 4 \mathrm{H}, \mathrm{J}_{\mathrm{H}-\mathrm{H}} 9, \mathrm{~N}_{-} \mathrm{C}_{6} \mathrm{H}_{4}-\mathrm{Cl}\right)$ 7.4-8.3 (m, 5H, $H$-Ar), $9.1(\mathrm{~s}, \mathrm{NH}) \mathrm{ppm} ;{ }^{13} \mathrm{C}$ NMR $\left(\mathrm{CDCl}_{3}\right): \delta=16.3\left(\mathrm{~d},{ }^{3} \mathrm{~J}_{C-P} 6.6\right.$, $2 \mathrm{CH}_{3}$ C.OP), 45.7 (d, $\left.{ }^{1} \mathrm{~J}_{C-P} 147, C \mathrm{H}-\mathrm{P}\right), 53.5\left(\mathrm{~d},{ }^{2} \mathrm{~J}_{C-P} 33, C \mathrm{H}-\mathrm{N}\right), 62.2\left(\mathrm{~d},{ }^{2} \mathrm{~J}_{C-P} 27\right.$, $2 \mathrm{CH}_{2}$.OP), 108.8, 115.3, 117.6, 124.9, 129.8, 130.1, 132.5, 139.9, 141.3, 144.8, $149.8,153.4,155.5(C=C, \mathrm{Ar}), 163.4\left(\mathrm{~d},{ }^{2} \mathrm{~J}_{C-P} 28, C=\mathrm{O}\right.$, ester), $185.7\left(\mathrm{~d},{ }^{4} \mathrm{~J}_{\mathrm{C}-\mathrm{P}} 4.6\right.$, $C=\mathrm{O}$, ring) ppm; ${ }^{31} \mathrm{P} \mathrm{NMR}\left(\mathrm{CDCl}_{3}\right): \delta=20.0 \mathrm{ppm} ; \mathrm{m} / z(\mathrm{EI})=493\left[\mathrm{M}^{+}\right](20)$.

[2-[(4-chlorophenyl)amino]-1- (ethoxycarbonyl)-2- (4-oxo-4H-chromen-3-yl) ethyl] diethyl-phosphonate (5b)

$5 \mathrm{~b}$ was obtained $(1: 1, \mathrm{v} / \mathrm{v})$ as brown crystals $40 \%$ m.p. $186-188{ }^{\circ} \mathrm{C}$ (from acetone) (Found: $\mathrm{C}, 56.31 ; \mathrm{H}, 5.16 ; \mathrm{Cl} ; 7.0 ; \mathrm{N}, 2.91 ; \mathrm{P}, 6.45 . \mathrm{C}_{24} \mathrm{H}_{27} \mathrm{ClNO}_{7} \mathrm{P}$ (507.92) requires $\mathrm{C}, 56.75 ; \mathrm{H}, 5.36 ; \mathrm{Cl}, 6.98 ; \mathrm{N}, 2.76 ; \mathrm{P}, 6.1) ; \mathrm{IR}(\mathrm{KBr}): \widetilde{v}=$ $1640\left(\mathrm{C}=\mathrm{O}\right.$, ring), $1720\left(\mathrm{C}=\mathrm{O}\right.$, ester), $1263(\mathrm{P}=\mathrm{O}), 1043(\mathrm{P}-\mathrm{O}-\mathrm{C}) ; 3240(\mathrm{NH}) \mathrm{cm}^{-}$ ${ }^{1} ;{ }^{1} \mathrm{H}$ NMR $\left(\mathrm{CDCl}_{3}\right): \delta=1.2\left(\mathrm{dt}, \mathrm{J}_{H-H} 7.9,{ }^{4} \mathrm{~J}_{H-P} 4.5,6 \mathrm{H}, 2 \mathrm{xH}_{3} \mathrm{CC} . \mathrm{OP}\right), 1.3(3 \mathrm{H}, \mathrm{t}$, $\mathrm{J}_{H-H} 7.1$, O-C.CH ${ }_{3}$, ester), 3.5 (dd, $\left.{ }^{2} \mathbf{J}_{H-P} 21,1 \mathrm{H}, \mathrm{CH}-\mathrm{P}\right), 4.3\left(\mathrm{dq}, \mathrm{J}_{H-H} 7.9^{3} \mathrm{~J}_{H-P} 5.4\right.$, $4 \mathrm{H}, 2 \mathrm{x} \mathrm{H}_{2} \mathrm{COP}$ ), 4.5 (q, $\mathrm{J}_{H-H} 7.4,2 \mathrm{H}, \mathrm{H}_{2} \mathrm{CO}$, ester), $5.1\left(\mathrm{dd},{ }^{3} \mathrm{~J}_{H-\mathrm{P}} 11.5,1 \mathrm{H}, \mathrm{CH}-\mathrm{N}\right)$, 6.5, 7.1 (2d, 4H, J $\left.\mathrm{J}_{H-H} 12.5, \mathrm{~N}_{-} \mathrm{C}_{6} \mathrm{H}_{4}-\mathrm{Cl}\right), 7.2-8.2(\mathrm{~m}, 5 \mathrm{H}, \mathrm{H}-\mathrm{Ar}), 9.1(1 \mathrm{H}, \mathrm{s}, \mathrm{NH})$ ppm; ${ }^{13} \mathrm{C}$ NMR $\left(\mathrm{CDCl}_{3}\right): \delta=14.3\left(\mathrm{CH}_{3}\right.$ C.O, ester $), 16.4\left(\mathrm{~d},{ }^{3} \mathrm{~J}_{C-P} 6.2,2 \mathrm{CH}_{3} \mathrm{C} . \mathrm{OP}\right)$, $42.5\left(\mathrm{~d},{ }^{1} \mathrm{~J}_{C-P} 145, C \mathrm{H}-\mathrm{P}\right), 53.2\left(\mathrm{~d},{ }^{2} \mathrm{~J}_{C-P} 33, C \mathrm{H}-\mathrm{N}\right), 61.6\left(\mathrm{~d},{ }^{4} \mathrm{~J}_{C-P} 3.8 C_{2} \cdot \mathrm{O}\right.$, ester), $62.3\left(\mathrm{~d},{ }^{2} \mathrm{~J}_{C-P} 35,2 \mathrm{CH}_{2} . \mathrm{OP}\right), 108.8,115.2,118.1,124.5,129.7,130.4$, $132.5,139.8,141.5,143.7,148.8,153.5,155.4(C=C, A r), 163.4\left(\mathrm{~d},{ }^{2} \mathrm{~J}_{C-P} 28\right.$, 
$C=\mathrm{O}$, ester), $185.7\left(\mathrm{~d},{ }^{4} \mathrm{~J}_{C-P} 4.6, C=\mathrm{O}\right.$, ring $) \mathrm{ppm} ;{ }^{31} \mathrm{P}$ NMR $\left(\mathrm{CDCl}_{3}\right): \delta=22.1$ $\mathrm{ppm} ; \mathrm{m} / z(\mathrm{EI})=507\left[\mathrm{M}^{+}\right](22)$.

[2-[(4-chlorophenyl) amino]-1-cyano-2- (4-oxo-4H-chromen-3-yl) ethyl] diethylphosphonate $(5 c)$

$5 \mathrm{c}$ was obtained $(1: 1, \mathrm{v} / \mathrm{v})$ as yellow crystals $40 \%$ m.p. $179-181{ }^{\circ} \mathrm{C}$ (from actone-ether) (Found : $\mathrm{C}, 57.12 ; \mathrm{H}, 4.55 ; \mathrm{Cl}, 7.71 ; \mathrm{N}, 5.98 ; \mathrm{P}, 6.45$; $\mathrm{C}_{22} \mathrm{H}_{22} \mathrm{ClN}_{2} \mathrm{O}_{5} \mathrm{P}$ (460.86), requires C, 57.34; $\left.\mathrm{H}, 4.81 ; \mathrm{Cl}, 7.69 ; \mathrm{N}, 6.08 ; \mathrm{P}, 6.72\right)$; IR (KBr): $\tilde{v}=1642(\mathrm{C}=\mathrm{O}$, ring), $2200(\mathrm{CN}), 1220(\mathrm{P}=\mathrm{O}), 1030(\mathrm{P}-\mathrm{O}-\mathrm{C}), 3240$ $(\mathrm{NH}) \mathrm{cm}^{-1} ;{ }^{1} \mathrm{H}$ NMR $\left(\mathrm{CDCl}_{3}\right): \delta=1.2\left(\mathrm{dt}, \mathrm{J}_{H-H} 7.9,{ }^{4} \mathrm{~J}_{H-P}\right.$ 4.6, $\left.6 \mathrm{H}, 2 \times \mathrm{H}_{3} \mathrm{CC} . \mathrm{OP}\right)$, $3.4\left(\mathrm{dd},{ }^{2} \mathrm{~J}_{H-P} 18,1 \mathrm{H}, \mathrm{CH}-\mathrm{P}\right), 4.2\left(\mathrm{dq}, \mathrm{J}_{H-H} 7.9{ }^{3} \mathrm{~J}_{H-P} 5.8,4 \mathrm{H}, 2 \times \mathrm{H}_{2} \mathrm{C} . \mathrm{OP}\right), 5.5(\mathrm{dd}$, $\left.{ }^{3} \mathrm{~J}_{H-P} 11.5,1 \mathrm{H}, \mathrm{CH}-\mathrm{N}\right), 6.6,7.1\left(2 \mathrm{~d}, \mathrm{~J}_{H-H} 12.6,4 \mathrm{H}, \mathrm{N}-\mathrm{C}_{6} H_{4}-\mathrm{Cl}\right), 7.3-8.3(\mathrm{~m}, 5 \mathrm{H}$, $H$-Ar), 9.5 (s, NH) ppm; ${ }^{13} \mathrm{C} \mathrm{NMR}\left(\mathrm{CDCl}_{3}\right): \delta=16.2\left(\mathrm{~d},{ }^{3} \mathrm{~J}_{C-P} 6.7,2 \mathrm{CH}_{3} \mathrm{C} . \mathrm{OP}\right)$, $32.5\left(\mathrm{~d},{ }^{1} \mathrm{~J}_{C-P} 147, C \mathrm{H}-\mathrm{P}\right), 52.4\left(\mathrm{~d},{ }^{2} \mathrm{~J}_{C-P} 30, C \mathrm{H}-\mathrm{N}\right), 61.6\left(\mathrm{~d},{ }^{2} \mathrm{~J}_{C-P} 35,2 \mathrm{CH}_{2} \mathrm{OP}\right)$, 115.2 (CN), 109.4, 115.3, 117.6, 122.1, 124.3, 129.5, 131.5, 133.4, 141.5, 143.7, 149.6, 153.8, $157.1(C=C, \mathrm{Ar}), 183.6\left(\mathrm{~d},{ }^{4} \mathrm{~J}_{C-P} 4.5, C=\mathrm{O}\right.$, ring); ppm; ${ }^{31} \mathrm{P}$ NMR $\left(\mathrm{CDCl}_{3}\right): \delta=20.2 \mathrm{ppm} ; \mathrm{m} / z(\mathrm{EI})=460\left[\mathrm{M}^{+}\right](18)$.

Methyl-4H -furo[3,2-c]chromene-2-carboxylate (8a)

8a was obtained (8:2, vlv) as yellow crystals, $26 \%$, m.p. $142-144{ }^{\circ} \mathrm{C}$ (From cyclohexane) (Found : $\mathrm{C}, 67.53 ; \mathrm{H}, 4.62 \mathrm{C}_{13} \mathrm{H}_{10} \mathrm{O}_{4}(230.22)$, requires $\mathrm{C}, 67.82, \mathrm{H}$, 4.38); IR (KBr): $\tilde{v}=1550(\mathrm{CH}=\mathrm{CH}), 1730(\mathrm{C}=\mathrm{O}) \mathrm{cm}^{-1} ;{ }^{1} \mathrm{H}$ NMR $\left(\mathrm{CDCl}_{3}\right): \delta=$ $3.7\left(\mathrm{~s}, 3 \mathrm{H}, \mathrm{CH}_{3} \mathrm{O}\right), 5.2\left(\mathrm{~s}, 2 \mathrm{H}, \mathrm{CH}_{2}\right.$-ring), 6.8-7.5 (m, 5H, $\left.\mathrm{H}-\mathrm{Ar}\right) \mathrm{ppm} ;{ }^{13} \mathrm{C} \mathrm{NMR}$ $\left(\mathrm{CDCl}_{3}\right): \delta=51.5\left(\mathrm{CH}_{3} \mathrm{O}\right), 65.5\left(\mathrm{CH}_{2}\right.$-ring $), 113.1,114.1,119.5,120.1,122.3$, 123.8, 129.6, 143.2, 144.5, $155.6(C=C, \mathrm{Ar}), 158.8(C=\mathrm{O}) ; \mathrm{ppm} ; \mathrm{m} / z(\mathrm{EI})=230$ $\left[\mathrm{M}^{+}\right]$(24).

Ethyl4H -furo[3,2-c] chromene-2carboxylate (8b)

8 b was obtained $\left(7: 3\right.$, vlv) as yellow crystals $28 \%$ m.p., $130-132{ }^{\circ} \mathrm{C}$ (From cyclohexane) (Found : C, 68.67; H, $4.72 \mathrm{C}_{14} \mathrm{H}_{12} \mathrm{O}_{4}(244.25)$, requires $\mathrm{C} 68.85, \mathrm{H}$, 4.95); IR (KBr): $\widetilde{v}=1590(\mathrm{CH}=\mathrm{CH}), 1740,(\mathrm{C}=\mathrm{O}) \mathrm{cm}^{-1} ;{ }^{1} \mathrm{H} \mathrm{NMR}\left(\mathrm{CDCl}_{3}\right): \delta=$ $1.3\left(\mathrm{t}, \mathrm{J}_{H-H} 7.5,3 \mathrm{H}, \mathrm{CH}_{3} \mathrm{C} . \mathrm{O}\right), 4.3\left(\mathrm{q}, \mathrm{J}_{H-H} 7.5,2 \mathrm{H}, \mathrm{CH}_{2} . \mathrm{O}\right), 5.0$ (s, $2 \mathrm{H}, \mathrm{CH}_{2}$.ring), 6.9-7.4 (m, 5H, H-Ar) ppm; ${ }^{13} \mathrm{C}$ NMR $\left(\mathrm{CDCl}_{3}\right): \delta=14.5\left(\mathrm{CH}_{3} \mathrm{C} . \mathrm{O}\right), 62.66$ $\left(\mathrm{CH}_{2} . \mathrm{O}\right)$, $64.6\left(\mathrm{CH}_{2}\right.$-ring $), 112.5,113.1,119.3,120.2,122.8,123.6,129.5,142.2$, 143.4, 155.6 ( $C=C, \mathrm{Ar}), 160.4(C=\mathrm{O}) ; \mathrm{ppm} ; \mathrm{m} / z(\mathrm{EI})=244\left[\mathrm{M}^{+}\right](18)$.

4H-furo [3,2-c] chromene-2-carbonitrile (8c)

$8 \mathrm{c}$ was obtained $(7: 3, \mathrm{vlv})$ as yellow crystals $25 \%$ m.p., $122-124{ }^{\circ} \mathrm{C}$ (From ether) (Found: C, 72.97; H, 3.19; N, 7.32 $\mathrm{C}_{12} \mathrm{H}_{7} \mathrm{NO}_{2}$ (197.196), requires C, 73.09; $\mathrm{H}, 3.58 ; \mathrm{N} ; 7.10)$; IR $(\mathrm{KBr}): \widetilde{v}=1600(\mathrm{CH}=\mathrm{CH}), 2220(\mathrm{CN}) \mathrm{cm}^{-1} ;{ }^{1} \mathrm{H}$ NMR $\left(\mathrm{CDCl}_{3}\right): \delta=4.9\left(\mathrm{~s}, 2 \mathrm{H}, \mathrm{CH}_{2}\right.$ - ring), 6.7-7.7 (m, 5H, H-Ar) ppm; ${ }^{13} \mathrm{C}$ NMR $\left(\mathrm{CDCl}_{3}\right): \delta=64.6\left(\mathrm{CH}_{2}\right.$-ring $), 110.4(\mathrm{CN}), 112.2,114.2,118.6,120.5,123.7$, $125.7,129.6,142.1,143.6,155.4(C=C, \mathrm{Ar}) ; \mathrm{ppm} ; \mathrm{m} / z(\mathrm{EI})=197\left[\mathrm{M}^{+}\right](25)$. 
Reaction of 3-(p-chlorophenyl-iminomethyl) chromone (1) with diethylvinylphosphonate (9): A preparation of compound 11

To a stirred solution of $1 \mathrm{~g}(3.5 \mathrm{mmol}) 1$ and $0.65 \mathrm{~g}(4 \mathrm{mmol}) 9 \mathrm{in} 30 \mathrm{ml} \mathrm{THF}$, freshly prepared $20 \mathrm{ml} \mathrm{LiOH}$ solution $(0.5 \mathrm{M})$ was added in one portion. The reaction mixture was stirred at r.t. for $2 \mathrm{hr}$ to ensure the complete dissolve of the reaction and then heated under reflux for $10 \mathrm{hr}$ (TLC). After removing the solvent; $20 \mathrm{ml}$ of dist. $\mathrm{H}_{2} \mathrm{O}$ was added and then extracted with $\mathrm{CHCl}_{3}$. After evaporation of the dried $\mathrm{CHCl}_{3}$ solution, the residue was chromatographed on silica gel using $\mathrm{n}$-hexane- $\mathrm{CDCl}_{3}$ as the eluents whereupon compound 11 and unidentified products were isolated.

3-[1-(4-chlorophenyl)-2-ethoxy-2-oxido-2,5-dihydro-1H-1,2-azaphosphol-5yl]-4H-chromen-4-one (11)

11 was obtaind $\left(1: 1\right.$, vlv) as pale brown crystals $68 \%$, m.p. $206-208{ }^{\circ} \mathrm{C}$ (From $\mathrm{CDCl}_{3}$ ) (Found: C, 59.62; H, 4.48; Cl, 8.65; N, 3.52; P, 7.55 $\mathrm{C}_{20} \mathrm{H}_{17} \mathrm{ClNO}_{4} \mathrm{P}(401.79)$ requires :C, 59.79; H, 4.26; $\mathrm{Cl}, 8.82 ; \mathrm{N}, 3.49 ; \mathrm{P}, 7.71) ; \operatorname{IR}(\mathrm{KBr}): \tilde{v}=1248(\mathrm{P}=\mathrm{O})$, $1098\left(\mathrm{P}_{-} \mathrm{OC}_{2} \mathrm{H}_{5}\right) \mathrm{cm}^{-1} ;{ }^{1} \mathrm{H} \mathrm{NMR}\left(\mathrm{CDCl}_{3}\right): \delta=1.42\left(\mathrm{dt},{ }_{H-H} 6.8,{ }^{4} \mathrm{~J}_{H-P} 4.4,3 \mathrm{H}\right.$, $H_{3}$ CC.OP), 3.8 (dq, $\left.\mathrm{J}_{H-H} 6.8,{ }^{3} \mathrm{~J}_{H-P} 5.6,2 \mathrm{H}, \mathrm{H}_{2} \mathrm{COP}\right), 5.0\left(\mathrm{~d},{ }^{3} \mathrm{~J}_{H-P} 5.2,1 \mathrm{H}, \mathrm{CH}-\mathrm{N}\right), 6.4$, $6.5\left(2 \mathrm{~d},{ }^{2} \mathrm{~J}_{H-P} 13.5,1 \mathrm{H}, \mathrm{CH}=\mathrm{CH}-\mathrm{P}\right), 7.5-8.48$ (m, $\left.10 \mathrm{H}, \mathrm{CH}=\mathrm{CH}-\mathrm{P}, \mathrm{H}-\mathrm{Ar}\right) \mathrm{ppm} ;{ }^{13} \mathrm{C}$ $\operatorname{NMR}\left(\mathrm{CDCl}_{3}\right): \delta=16.4\left(\mathrm{~d},{ }^{3} \mathrm{~J}_{C-P} 6.4, \mathrm{H}_{3}\right.$ CC.OP), $60.7\left(\mathrm{~d},{ }^{2} \mathrm{~J}_{C-P} 37, \mathrm{H}_{2}\right.$ CO.P), $59.7(\mathrm{~d}$, $\left.{ }^{3} \mathrm{~J}_{C-P} 5.5, C \mathrm{H}-\mathrm{N}\right), 107.0,10.2,117.1,119.0,121.7,124.0,124.3,127.8,128.1,133.3$, 138.4, 153.4, 155.2, 157.5( $C=C$, phenyl), $178.4(C=\mathrm{O}) \mathrm{ppm} ;{ }^{31} \mathrm{P} \mathrm{NMR}\left(\mathrm{CDCl}_{3}\right): \delta=$ $12.2 \mathrm{ppm} ; \mathrm{m} / \mathrm{z}(\mathrm{EI})=401\left[\mathrm{M}^{+}\right](13)$.

Reaction of 1 with cyanomethylenetriphenylphosphonium chloride (12): A preparation of compound 14

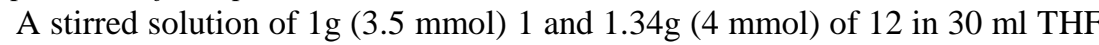
was treated with $10 \mathrm{ml}$ of aqueous $\mathrm{LiOH}$ solution $(0.5 \mathrm{M})$ and the mixture was heated under reflux for $8 \mathrm{hr}$. The reaction mixture was worked up as described for the reaction $1+9$, and separated by column chromatography, using n-hexaneAcOEt as eluents yielding compound 14 .

3-[(4-chlorophenyl)amino]-3-(4-oxo-4H-chromen-3-yl)propanenitrile (14)

14 was obtained $\left(7: 3\right.$, vlv) as yellow crystals $58 \%$ m.p. $180-182{ }^{\circ} \mathrm{C}$ (from $\mathrm{CH}_{2} \mathrm{Cl}_{2}$ ) (Found: C, 66.32; $\mathrm{H}, 3.99 ; \mathrm{Cl}, 10.81 ; \mathrm{N}, 8.42 \mathrm{C}_{18} \mathrm{H}_{13} \mathrm{ClN}_{2} \mathrm{O}_{2}$ (324.77) requires $\mathrm{C}, 66.57 ; \mathrm{H}, 4.03 ; \mathrm{Cl}, 10.92 ; \mathrm{N}, 8.63) ; \mathrm{IR}(\mathrm{KBr}): \tilde{v}=3260(\mathrm{NH}), 2200$ $(\mathrm{CN}) \mathrm{cm}^{-1} ;{ }^{1} \mathrm{H} \mathrm{NMR}\left(\mathrm{CDCl}_{3}\right): \delta=3.9,4.0\left(2 \mathrm{~d}, \mathrm{~J}_{H-H} 6.8,2 \mathrm{H}, \mathrm{CH}_{2}-\mathrm{CN}\right), 5.1\left(\mathrm{t}, \mathrm{J}_{H-H} 6.8\right.$, $\left.1 \mathrm{H}, \mathrm{CH}-\mathrm{CH}_{2}\right), 6.6-8.2(\mathrm{~m}, 9 \mathrm{H}, H-\mathrm{Ar}), 9.4(\mathrm{~s}, 1 \mathrm{H}, \mathrm{NH}) \mathrm{ppm} ;{ }^{13} \mathrm{C} \mathrm{NMR}\left(\mathrm{CDCl}_{3}\right): \delta=$ $23.3\left(\mathrm{CH}_{2}\right), 47.4(\mathrm{CH}), 114.6(\mathrm{CN}), 107.1,110.7,116.2,117.0,121.6,121.8,124.0$, $127.8,129.0,133.3,151.4,155.3,157.9(C=C$, phenyl $), 180.3(\mathrm{C}=\mathrm{O}) \mathrm{ppm} ; \mathrm{m} / \mathrm{z}(\mathrm{EI})=$ $324\left[\mathrm{M}^{+}\right](16)$.

Reaction of 1 with allyltriphenylphophhonium bromide (15): A preparation of compound 17

A solution of $1.35 \mathrm{~g}$ phophonium bromide 15 and $1 \mathrm{~g}$ of chromone 1 in $30 \mathrm{ml}$ THF was treated with $10 \mathrm{ml}$ of aqueous $\mathrm{LiOH}(0.5 \mathrm{M})$ for $1 \mathrm{hr}$ at room temperature, and then refluxed for $5 \mathrm{hr}$. The reaction mixture was worked up as Egypt. J. Chem. 53, No.5 (2010) 
described for the reaction $1+9$ and separated by column chromatography using $n$ hexane-AcOEt as eluents yielding 17.

3-\{1-[(4-chlorophenyl)amino]-2-methylprop-2-en-1-yl\}-4H-chromen-4-one (17) was obtained $(1: 1, \mathrm{v} / \mathrm{v})$ as yellow crystals $60 \%$, m.p. $220-222{ }^{\circ} \mathrm{C}$ (from $\mathrm{CH}_{2} \mathrm{Cl}_{2}$ ) (Found : C, 70.31; H, 5.01; Cl, 10.76; N, $4.52 \mathrm{C}_{19} \mathrm{H}_{16} \mathrm{ClNO}_{2}$ (325.79) requires: $\mathrm{C}, 70.05 ; \mathrm{H}, 4.95 ; \mathrm{Cl}, 10.88 ; \mathrm{N}, 4.30) ; \mathrm{IR}(\mathrm{KBr}): \tilde{v}=1645(\mathrm{C}=\mathrm{O}$, ring), $3200(\mathrm{NH}) \mathrm{cm}^{-1} ;{ }^{1} \mathrm{H}$ NMR $\left(\mathrm{CDCl}_{3}\right): \delta=1.9\left(\mathrm{~s}, 3 \mathrm{H}, \mathrm{CH}_{3}\right), 4.8,5.1\left(2 \mathrm{~d}, \mathrm{~J}_{H-H} 1.8\right.$, $\left.2 \mathrm{H}, \mathrm{CH}_{2}=\mathrm{C}\right), 5.5(\mathrm{~s}, 1 \mathrm{H}, \mathrm{CH}-\mathrm{N}), 6.5-8.2(\mathrm{~m}, 9 \mathrm{H}, H-\mathrm{Ar}), 9.3(\mathrm{~s}, 1 \mathrm{H}, \mathrm{NH}) \mathrm{ppm} ;{ }^{13} \mathrm{C}$ NMR $\left(\mathrm{CDCl}_{3}\right): \delta=23.0\left(\mathrm{CH}_{3}\right), 56.1(\mathrm{CH}), 109.3,114.7,116.2,119.2,120.5$, $121.8,124,125.5,128.7,133.3,150.7,151.6,152.6,155.7$ ( $C=C$, phenyl), 181.7 $(C=\mathrm{O}) \mathrm{ppm} ; \mathrm{m} / \mathrm{z}(\mathrm{EI})=325\left[\mathrm{M}^{+}\right](21)$.

\section{References}

1. (a) Boulos, L. S. and Yakout, El-Sayed M. A., The behavior of 5- (4-pyridyl)-2thiono-1,3,4-oxadioazole towards Wittig-Horner reagents. Phosphorus, Sulfur and Silicon 84, 35(1993).(b) Abdou, W. M. and Khidre, M. D., fused phosphono substituted $\mathrm{O}$ - and $\mathrm{N}$-heterocycles via condensative cyclisation reaction of $\alpha$ phosphonyl carbanions with 4-thiazolidinones. Phosphorus, Sulfur and Silicon and the Related Elements 179, 1307 (2004).

2. (a) Boulos, L. S. and Arsanious, M. H. N., The reaction of Wadsworth-EmmonsHorner reagents with O-and P-quinoneimines.Phosphorus, Sulfur and Silicon, 89, 185 (1994). (b) Abdou, W. M., Khidre, M. D., Khidre, R. E., Diverse reactivity of $\alpha$ Carbanions Derived from Alkylidenephosph-oranes toward 2-(1 $\lambda 5$-diazynylidene)1H-indene-1,3-(2H)dione. General approach to conjugated oxadiazines, pyridazines and Spiro [3]pyrazoles. J. Heterocycle Chem. 45, 1571 (2008).

3. (a) Boulos, L. S., Arsanious, M. H. N. and Eldin, N. K., The reaction of WittigHorner reagents and phosphacumulenes with nitroso naphthol. Phosphorus, Sulfur and Silicon, 122, 49 (1997). (b) Khidre, M. D., Kamel, A. A. and Abdou, W. M., An approach to biologically important cyclic, acyclic $S$ - compounds and their phosphono derivatives, J. Heterocyclic Chem. 42, 103 (2005).

4. (a) Boulos, L. S., Arsanious, M. H. N., Tetrahedron 53, (1997), 3649. (b) Abdou, W. M., Khidre, M. D. and Kamel, A. A., Similarity and dissimilarity between Wittig- and Wittig-Horner synthon. Reactivity toward cyclic and acyclic cis-disulfides. Heterocyclic Comms. 10, 217 (2004)

5. Yakout, El-Sayed M. A., Giurgius, D. B. and Boulos, L. S., The behvior of 6methyl-2-thiouracil towards Wittig-Horner reagents. Phosphorus, Sulfur and Silicon, 148, 177(1999). 
6. Boulos, L. S. and Abd-El-Malek, H. A., The reaction of wittig and Wittig-Horner reagents with dicyanomethylene derivatives of fluorenone, xanthone and thiaxanthone. A Novel synthesis of phosphoranylidene derivatives. Heteroatom Chem. 10, 263(1999).

7. Abdou, W. M., Salem, M. A. I. and Barghash, R. F., New phosphono substituted 3and 5-membered rings starting from 3(2-Thienyl)acrylonitriles and - phosphoryl Carbanions. Synth. Communs. 33, 1341(2003).

8. Khidre, M.D., Abou-yousef, H. M. and Mahran, M. R. H., On the reaction of 3(aryliminomethyl) chromones with alkyl phosphites and methylenetriphenlphosphoranes (Wittig Reagents). Phosphorus, Sulfur and Silicon, 140, 147(1998).

9. Crutchfield, M. M., Dungan, O. H., Letcher, J. H., Mark, V. and van Wazer, J. R., In: Topics in Phosphorus Cemistry, (1967) Vol. 5, Inter-Science Publishers; New York, pp. 227-447.

10. Ramirez, F., Madan, O. P. and Smith, C. P., Tetrahedron Lett. 3, 201(1965).

11. Silverstein, R.M., Bassler, G.C. and Morril, T.C., (Ed.) Spectroscopic Identification of Organic Compounds, John Wiley and Sons. Inc., New York, $4^{\text {th }}$ Edn. 1981.

12. Boulos, L. S. and Yakout, El- Sayed M. A., Heteroatom Chem. 8, 253 (1997).

13. Johnson, A. Wm., Ylides and Imines of Phosphorus, John Wiley and Sons, New York, pp. 153-220 (1993).

14. (a) Abdou, W. M., Shaddy, A. A. and Sediek, A. A., Antimicrobial activity of novel fused nitrogen, sulfur and posphorus containing- heterocycles and relevant phosphonates with difurylpyridazine species.Chemical Research, 8 (2009), (b) Abdou, W. M., Khidre, M. D. and Khidre, R. E., Application of phosphonyl carbanions to highly regioselective synthesis of some diazaphospholes and pyrazolinyl phosphonates.European Journal of Medicinal Chemistry, 1(2008),(c) Bhuran Kumar, N. N., Nagarjuna Reddy, M. and Kumara Swamy, K.C., J. Reactivity of allenylphosphonates toward salicylaldehydes and activated phenols: Facile synthesis of chromenes and substited butadienes. J. Org. Chem. 74, 5395(2009) (d) Shi, Y. L. and Minshi, The Synthesis of chromenes, chromanes, coumarins and related heterocycles via tandem reactions of Sslicylic aldehydes or salicylic imines with $\alpha, \beta$ unsaturated compounds. Org. Biomol. Chem. 5, 1499 (2007).

15. Abdou, W. M., Ganoub, N. A. F., Fahmy, A. F. M. and Shaddy, A. A. M., Regioselective condensation of alkylidenephosphoranes with bifunctionalized compounds: New approach to the synsthesis of fused O- and N-Heterocycles. Phosphorus, Sulfur and Silicon, 180, 2373 (2004).

Egypt. J. Chem. 53, No.5 (2010) 
16. (a) Croce, P. D. and Pocar, D. J., J. Chem. Soc., Perkin Trans. I 619 (1976) (b) Zbiral, E., Reaktionen mit phosphororganischen verbindungen zur Reaktivitat von Bacylvinylphosphonium salzen ein neuartiges bildungsprinzip des thiazolringsy Tetrahedron Lett. (1970), 5107. (c) Abdou,W. M. and Kamel, A.A. A remarkably efficient and direct route to quinolines and benzoazepines from the condensation of Benzoxazinediones with Phosphonium Carbanion Salts. Synthetic Communications 37, 3945 (2007) (d) Abdou, W. M., Ganoub, N., Fahmy, A. F. M. and Shaddy, A. A. M., Heteroatom Chem. 16, 56 (2005).

17. Fitton, A. O., Frost, J. R. and Houghton, P. G. J. Chem. Soc., Perkin Trans. 1, 1450 (1977)

(Received 7/ 4/ 2010 ;

accepted 15/9/2010) 


\section{دراسة تكميلية لنشاط r-p-كلوروفنيل-أمينوميثل كرومون نحو كواشف الفسفور}

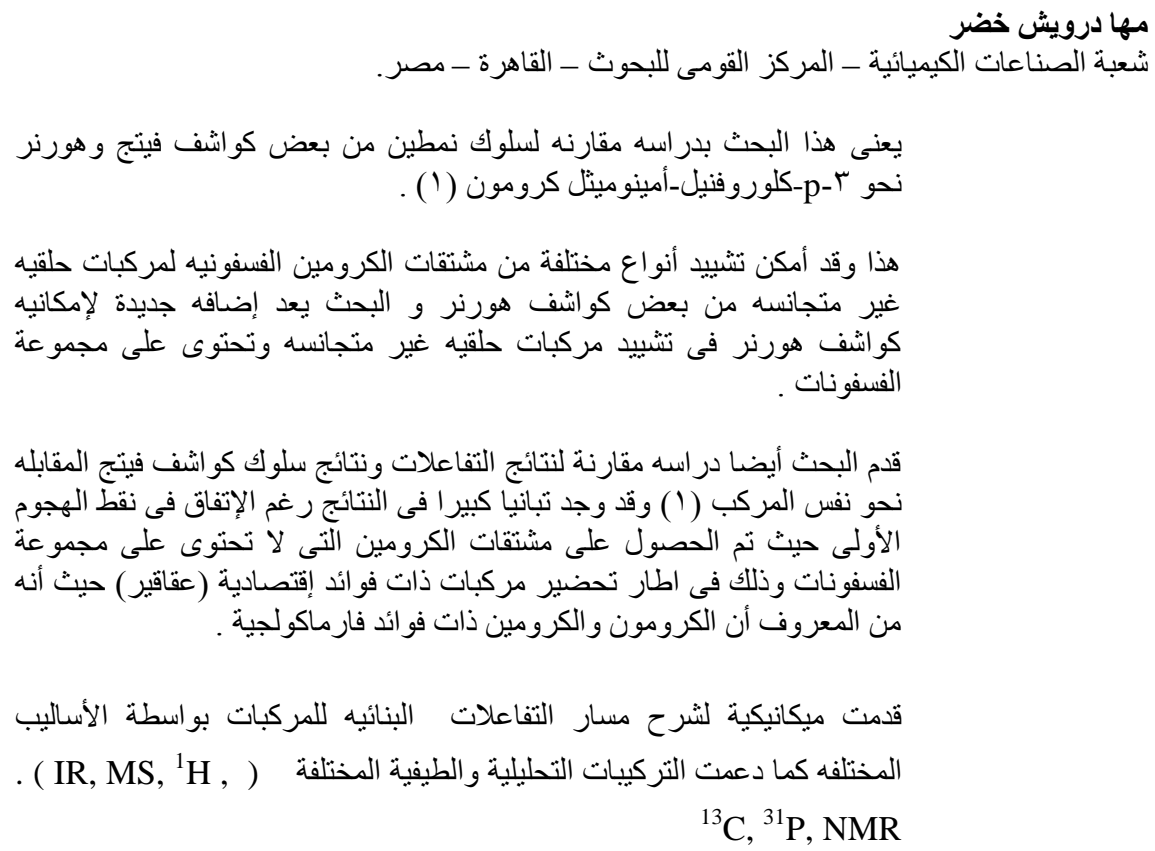

